NICU for a suspected infection (defined by clinical or analytical sepsis, fever without a source, or neurological symptoms) are included in the study. Neonates with clear respiratory or gastrointestinal symptoms are excluded. A screening for bacterial and viral infection is performed. In negative cases, a real-time-RT-PCR for HPeV detection is performed in cerebrospinal fluids.

Results We present the data for the first 18 months of the study period. 28 newborns were included in the study. There were 2 confirmed cases of HPeV-3 infection. Both presented in May 2013, with fever of unknown origin, and had normal cerebrospinal fluid analysis. Case 1 was 6 days old. Case 2 was 19 days old and during the hospitalisation period developed diarrhoea and respiratory apneas requiring oxygen support. Both had a favourable evolution.

Conclusions Clinicians should include $\mathrm{HPeV}$ in the differential diagnosis of neonatal infection.

\section{PS-306 NEONATAL LUMBAR PUNCTURE - ARE CLINICAL LANDMARKS ACCURATE?}

${ }^{1}$ BJW Baxter, ${ }^{1} \mathrm{~J}$ Evans, ${ }^{1} \mathrm{R}$ Morris, ${ }^{1} \mathrm{U}$ Ghafoor, ${ }^{1} \mathrm{M}$ Nana, ${ }^{1} \mathrm{~T}$ Weldon, ${ }^{2} \mathrm{G}$ Tudor, ${ }^{1} T$ Hildebrandt. ${ }^{1}$ Paediatrics and Neonatology, Princess of Wales Hospital, Bridgend, UK; ${ }^{2}$ Paediatric Radiology, Princess of Wales Hospital, Bridgend, UK

\subsection{6/archdischild-2014-307384.605}

Background The intercristal line, defined by the superior aspect of the iliac crest, is used to clinically identify the correct position for lumbar puncture in neonates. Accepted practice is to insert the needle in the intervertebral space at L3/4 or L4/5.

Aim We hypothesised variation in anatomical structures in term neonates and that the intercristal line might not accurately aid identification of the correct intervertebral space.

Method Following ethical approval 30 term neonates were recruited. Paediatricians identified and marked the intercristal line and intervertebral space above, with neonates in left lateral position. The anatomical position of both points and the conus medullaris end point were confirmed using ultrasonography.

Results The intercristal line was marked from L2/3 to L5/S1. In 25 neonates $(83 \%)$ the intercristal line was identified between $\mathrm{L} 3 / 4$ and $\mathrm{L} 4 / 5$. The intervertebral space above this line was marked between L1/2 to L4/5. The potential site for lumbar puncture was identified too high in 11 cases (36\%).

The end of the conus medullaris ranged from L1 to L3 terminating at L2 or lower in 11 cases (36\%).

Conclusion There is wide variation in the position of the intercristal line and potential lumbar puncture site. The potential lumbar puncture site was marked higher than anticipated at L2/3 or above in 11 neonates (36\%). The end of the conus medullaris was identified in 11 neonates (36\%) at L2 or lower. Using the intercristal line to guide lumbar puncture does not appear to be accurate raising the possibility of potential spinal cord damage.

\section{PS-307 NATIONAL RECOMMENDATIONS FOR THE INVESTIGATION OF EARLY ONSET NEONATAL SEPSIS: LUMBAR PUNCTURE INCIDENCE AND INDICATIONS}

R Irving, P Lister, D Jolley, LF Hamilton, HE Gillet, JW Davis. Neonatology, St Michael's Hospital University Hospitals Bristol, Bristol, UK

\subsection{6/archdischild-2014-307384.606}

Background Early onset sepsis (EOS) is a leading cause of morbidity and mortality in newborns. The incidence of culture- positive EOS in UK neonates is 0.9/1000 live births. We aimed to compare EOS investigation practice with The National Institute of Clinical Excellence (NICE) guidelines (2012) for investigation, and determine factors that influence LP acquisition.

Method We conducted a retrospective observational study in a regional neonatal unit, using data from 213 babies investigated for EOS $(<48 \mathrm{~h})$. We reviewed patient notes, electronic records, and pathology results in 2 epochs (September-October 2012, April-May 2013). Clinical concern of infection was judged retrospectively using NICE criteria (any 'Red Flag', $>2$ risk factors, or other clinical indicators).

Results Data was collected from 213 term babies (228 infection episodes). Clinical concern for infection was identified in 52/ 228. These infants had more respiratory distress and shock than those without clinical concern. $(p<0.05)$. In total 31/228 babies had an LP; 14/31 infants had clinical concern of infection. Clinical concern for infection was similar in the 'LP group' and 'nonLP group'. The 'LP group' had a larger proportion of maternal pyrexia $(18 / 31$ vs. $60 / 137, \mathrm{p}<0.05)$ and median maximum CRP (19 mg/l vs. $1 \mathrm{mg} / \mathrm{l}, \mathrm{p}<0.05)$ than the 'non-LP group'. There were no positive blood/CSF cultures.

Conclusion Despite its low incidence EOS causes substantial clinical concern. LPs were not carried out in a number of infants despite meeting NICE criteria. Maternal risk factors and maximum CRP appear to determine the acquisition of LP more than physician 'concern' of infection.

\section{PS-308 NEONATAL BACTERIAL MENINGITIS IN A DEVELOPING COUNTRY}

F Kamoun, S Loukil, L Sfaihi, S Ben Ameur, TH Kamoun, M Hachicha. Pediatrics Department, Hedi Chaker Hospital, Sfax, Tunisia

10.1136/archdischild-2014-307384.607

Background and aims Neonatal bacterial meningitis (NBM) is a serious disease with high morbidity and mortality rates.

Aims Study the epidemiological, clinical, bacteriological aspects and the outcome of NBM.

Methods We report a retrospective analysis of 55 cases of NBM hospitalised in the paediatric department between 1990 and 2012. Inclusion criteria were infants less than 29 days of age who were hospitalised for bacterial meningitis diagnosed on either the presence of bacteria in cerebrospinal fluid (CSF) or with more than 30 cells $/ \mathrm{mm}^{3}$, predominance of neutrophils, the protein level greater than $1.2 \mathrm{~g} / \mathrm{l}$ and hypoglycorachia.

Results The mean age of diagnosis was 11 days and the sex ratio was 1.75 . The patients were premature in $9 \%$ and low birth weight in $20 \%$ of cases. The main circumstances of discovery were fever (69.1\%), refusal to breastfeed (49.1\%) and seizures (16.4\%). A myelomeninguolcele was present in 6 patients. Blood culture was positive in $34.8 \%$ of cases and the CSF culture was positive in $54.4 \%$ of cases. The main bacteria isolated was Escherichia coli (7 cases), Streptococcus B (7 cases) and Pseudomonas aeruginosa (5 cases). The cefotaximeampicillingentamicin combination was the most prescribed first-line. Ofloxacin was associated initial antibiotic therapy in 9 cases. The mortality rate was $40 \%$ and the sequelae rate in survivors was $27 \%$.

Conclusion This study emphasises the severity of NBM with high rates of mortality and neurological sequelae. An early diagnosis and effective antibiotic therapy is needed to improve the prognosis. 